\title{
Human mast cell neutral proteases generate modified LDL particles with increased proteoglycan binding
}

\author{
Katariina Maaninka a, Su Duy Nguyen a, Mikko I. Mäyränpää a, b, Riia Plihtari a ,

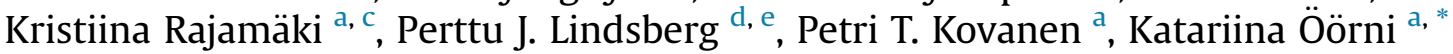 \\ ${ }^{a}$ Wihuri Research Institute, Biomedicum 1, Haartmaninkatu 8, 00290 Helsinki, Finland \\ ${ }^{\mathrm{b}}$ Pathology, University of Helsinki and Helsinki University Hospital, Finland \\ c Clinicum Department, Faculty of Medicine, University of Helsinki, Helsinki, Finland \\ d Molecular Neurology, Research Programs Unit, University of Helsinki, Helsinki, Finland \\ e Clinical Neurosciences, Neurology, University of Helsinki and Helsinki University Hospital, Finland
}

\section{A R T I C L E I N F O}

\section{Article history:}

Received 27 October 2017

Received in revised form

6 March 2018

Accepted 12 April 2018

Available online $\mathrm{xxx}$

\section{Keywords:}

(3-7) аров

Lipoprotein

LDL

Mast cell

Cathepsin G

Tryptase

Proteoglycan

\begin{abstract}
A B S T R A C $T$
Background and aims: Subendothelial interaction of LDL with extracellular matrix drives atherogenesis. This interaction can be strengthened by proteolytic modification of LDL. Mast cells (MCs) are present in atherosclerotic lesions, and upon activation, they degranulate and release a variety of neutral proteases. Here we studied the ability of MC proteases to cleave apoB-100 of LDL and affect the binding of LDL to proteoglycans.

Methods: Mature human MCs were differentiated from human peripheral blood-derived CD34 ${ }^{+}$progenitors in vitro and activated with calcium ionophore to generate MC-conditioned medium. LDL was incubated in the MC-conditioned medium or with individual MC proteases, and the binding of native and modified LDL to isolated human aortic proteoglycans or to human atherosclerotic plaques ex vivo was determined. MC proteases in atherosclerotic human coronary artery lesions were detected by immunofluorescence and qPCR.

Results: Activated human MCs released the neutral proteases tryptase, chymase, carboxypeptidase A3, cathepsin G, and granzyme B. Of these, cathepsin G degraded most efficiently apoB-100, induced LDL fusion, and enhanced binding of LDL to isolated human aortic proteoglycans and human atherosclerotic lesions ex vivo. Double immunofluoresence staining of human atherosclerotic coronary arteries for tryptase and cathepsin G indicated that lesional MCs contain cathepsin G. In the lesions, expression of cathepsin $\mathrm{G}$ correlated with the expression of tryptase and chymase, but not with that of neutrophil proteinase 3 .

Conclusions: The present study suggests that cathepsin G in human atherosclerotic lesions is largely derived from MCs and that activated MCs may contribute to atherogenesis by enhancing LDL retention.
\end{abstract} (C) 2018 Elsevier B.V. All rights reserved.

\section{Introduction}

Atherogenesis is initiated at atherosclerosis-susceptible sites of the arterial wall by focal retention of LDL particles within the subendothelial extracellular matrix (ECM), particularly via binding of LDL to proteoglycans (PGs) [1]. Indeed, the atherogenicity of LDL is directly linked to its affinity for arterial wall PGs [1-3], which is related to either the properties of LDL or the PGs. The PG-LDL

\footnotetext{
* Corresponding author. Wihuri Research Institute, Biomedicum Helsinki, Haartmaninkatu 8, FI-00290 Helsinki, Finland.

E-mail address: kati.oorni@wri.fi (K. Öörni).
}

interaction is ionic and takes place between clusters of positively charged amino acid residues on apoB-100 and negatively charged sulfate and carboxyl groups on the glycosaminoglycan chains of PGs [4]. The principal site in apoB-100, which mediates the PG binding of LDL spans the amino acid residues 3359-3369 (referred to as site B) [2]. However, LDL particles seem to contain additional PGbinding sites as indicated by identification of several regions in apoB-100 that bind glycosaminoglycans, [5]; yet, these binding sites may not be functionally available on native LDL particles [2]. Importantly, various modifications of LDL particles have been shown to increase the binding of LDL to PGs [6-8], implying that additional PG-binding sites may become functional when LDL is 
modified $[9,10]$.

In the arterial intima, LDL particles are subjected to various structural and conformational modifications that promote the formation of LDL-derived lipid droplets and vesicles in the subendothelial extracellular space [7]. Such extracellular lipid particles isolated from human atherosclerotic lesions have features revealing that the apoB-100-containing plasma lipoproteins, which have entered the subendothelial space, have been exposed to proteolysis and lipolysis [11]. Indeed, atherosclerotic lesions contain several proteases capable of hydrolyzing the apoB-100 of LDL in vitro, including both plasma-derived and locally synthesized proteases. To the former group belong plasmin, kallikrein, and thrombin, and to the latter group the lysosomal proteases cathepsins D, F, K, and S secreted by intimal macrophages and smooth muscle cells of the lesions [6,12-14].

An additional source of proteolytic enzymes in the arterial intima are mast cells (MCs). This type of inflammatory cell is present in both early and advanced human atherosclerotic lesions [15-17]. The cytoplasm of MCs is filled with secretory granules, which contain high amounts of various neutral proteases [18]. All MCs in atherosclerotic lesions contain tryptase, and a variable fraction contain also chymase [15-17], both of which are virtually MCspecific proteases [19]. A third major neutral granule protease of MCs is carboxypeptidase A3 (CPA3), a zinc-dependent exopeptidase, which has previously been thought to be exclusively expressed in MCs; however, more recently transcripts of CPA3 have been found also in basophils [19]. In addition, human MC neutral granule proteases include two serine proteases that are not unique to this cell type, namely cathepsin G and granzyme B [20-22]. When MCs are activated, large amounts of the granule proteases are released into the extracellular surroundings in a process called degranulation. Unlike most other secreted proteases, the MC granule proteases are stored in fully active form, and, thus, once released they are capable of immediately triggering proteolytic events in the extracellular microenvironment of the tissue in which the activated MCs are present and degranulation occurs.

Recently, we developed a cell culture method for the differentiation of mature MCs from their circulating progenitor cells [23], and showed that mature MCs expressed a wide spectrum of the known MC neutral proteases, namely the tryptases derived from the TPSAB1, TPSB2, TPSD1, and TPSG1/PRSS31 genes, and chymase, CPA3, cathepsin G, and granzyme B [21]. In the present study, we investigated the ability of these neutral proteases to cleave apoB100 of LDL, and determined the effects of such proteolysis on the binding of LDL to human aortic PGs.

\section{Materials and methods}

\subsection{Cell preparation and culture}

Human MCs were in vitro differentiated from buffy coats of healthy volunteers, as previously described [23]. The buffy coats were supplied by the Finnish Red Cross Blood Service (Helsinki, Finland) with the approval of the Ethics Committee of the Finnish Red Cross.

\subsection{Preparation of mast cell-conditioned medium (mast cell releasate)}

At week 9 of culture, MCs at a concentration of $2 \times 10^{6} \mathrm{MCs} / \mathrm{ml}$ were activated by $1 \mu \mathrm{M}$ calcium ionophore A21387 (Sigma-Aldrich) in Dulbecco's phosphate buffered saline (DPBS) in a humidified incubator at $37^{\circ} \mathrm{C}\left(5 \% \mathrm{CO}_{2}\right)$. After $30 \mathrm{~min}$ of incubation, the MCs were sedimented, and the supernatant containing the released neutral proteases, i.e the MC-conditioned medium, which will be referred to as the $\mathrm{MC}$ releasate, was collected and stored at $-80^{\circ} \mathrm{C}$ until further analysis.

\subsection{Protease activity assays and protease inhibitors}

The amounts of active neutral proteases in MC releasates were determined through hydrolysis of specific protease substrates, as previously described [21]. In some experiments, some of the proteases in the $\mathrm{MC}$ releasates were inhibited as follows: tryptase with $200 \mu \mathrm{g} / \mathrm{ml}$ leupeptin (Sigma-Aldrich), chymase with $670 \mathrm{nM}$ diphenyl Na-benzoxycarbonyl-L-Arg-Glu-Thr-PheP-phosphonate [(RETF- $(\mathrm{OPh})_{2}$ ] generously provided by Dr Gunnar Pejler (Uppsala University, Uppsala, Sweden), and CPA3 with $1 \mu \mathrm{l} / 100 \mu \mathrm{l}$ Carboxypeptidase Inhibitor from potato tuber (Sigma-Aldrich).

\subsection{Preparation of human aortic proteoglycans}

PGs from the intima-media of human aortas were obtained at autopsy within $24 \mathrm{~h}$ of accidental death and prepared as described previously [24,25]. The Finnish National Authority for Medicolegal Affairs had approved the study. The amounts of the PGs are expressed in terms of their glycosaminoglycan content, as determined by the method of Bartold and Page [26].

\subsection{Isolation and modifications of $L D L$}

Human LDL $(\mathrm{d}=1.019-1.050 \mathrm{~g} / \mathrm{ml})$ were isolated from plasma of healthy volunteers (supplied by the Finnish Red Cross Blood Service) by sequential ultracentrifugation in the presence of $3 \mathrm{mM}$ EDTA, as described $[27,28]$. The amount of LDL is expressed in terms of its protein content determined by the method of Lowry and/or BCA Protein Assay Kit (Thermo Scientific), with bovine serum albumin as standard. MC protease-modified LDL was prepared by incubation of LDL ( 1 or $2 \mathrm{mg} / \mathrm{ml}$ ) either with MC releasate, or separately with $1.5 \mu \mathrm{g} / \mathrm{ml}$ recombinant human chymase (SigmaAldrich), $20 \mu \mathrm{g} / \mathrm{ml}$ recombinant human $\beta$-tryptase (a kind gift from Dr. Christian Sommerhoff), $4.5 \mu \mathrm{g} / \mathrm{ml}$ cathepsin $\mathrm{G}$ from human neutrophils (Biomol), $10 \mu \mathrm{g} / \mathrm{ml}$ CPA from bovine pancreas (SigmaAldrich), or $0.1 \mu \mathrm{g} / \mathrm{ml}$ granzyme B (QuickZyme Biosciences) in DPBS overnight at $37^{\circ} \mathrm{C}$. The protease concentrations were chosen based on those found in the $\mathrm{MC}$ releasates. In some experiments, the apoB-100 of LDL was labeled before treatment with MC neutral proteases with a ${ }^{3} \mathrm{H}$-labeling reagent (t-butoxycarbonyl-L- $\left[{ }^{3} \mathrm{H}\right]$ methionine $N$-hydroxy-succinimidyl ester [Amersham Biosciences]) according to the Bolton-Hunter procedure [29].

The degree of apoB-100 proteolysis was determined by measuring the amount of radioactive peptides released from the LDL particle using the trichloroacetic acid (TCA) precipitation method [30], or by measuring the amount of immunoreactive apoB-100 with apoB ELISA (Mabthech) according to the manufacturer's instructions. SDS-PAGE was performed under denaturing conditions using Novex Bolt Bis-Tris Plus 4-12\% precast minigels and Bolt MES-SDS Running buffer (both Invitrogen, Thermo Fisher Scientific) according to the manufacturer's instructions. The extent of oxidation of LDL was determined by measuring the amount of thiobarbituric acid reactive substances (TBARS) [31].

In some experiments plasma of three healthy donors was pooled prior to LDL isolation. The pooled LDL $(1 \mathrm{mg} / \mathrm{ml})$ was then incubated overnight with the recombinant human chymase $(1.5 \mu \mathrm{g} /$ $\mathrm{ml})$ or with cathepsin $\mathrm{G}(4.5 \mu \mathrm{g} / \mathrm{ml})$ in the presence of human aortic PGs $(100 \mu \mathrm{g} / \mathrm{ml})$, after which cleavage of apoB-100 of LDL was visualized by SDS-PAGE. Finally, a fraction of the pooled LDL was conjugated with Atto 594 N-Hydroxysuccinimide (NHS) ester fluorochrome (Sigma Aldrich), as previously described [32], with the exception of using $0.2 \mathrm{M} \mathrm{NaHCO}_{3}$. 


\subsection{Binding of $L D L$ to human aortic proteoglycans}

Wells of 96-well plates were coated with $100 \mu \mathrm{l}$ of PGs dissolved in DPBS (at $25 \mu \mathrm{g} / \mathrm{ml}$ ) by incubation at $4{ }^{\circ} \mathrm{C}$ overnight. Thereafter, the wells were blocked with $250 \mu$ l of buffer containing $3 \%$ BSA, $1 \%$ fat-free milk, and $0.05 \%$ Tween-20 in DPBS for 60 min at $37^{\circ} \mathrm{C}$. Noncoated wells incubated with blocking buffer only served as controls.

After washing of the wells, $2 \mu \mathrm{g}$ LDL that had been modified with MC releasate, or alternatively with individual neutral proteases, was added to the wells in a total volume of $100 \mu \mathrm{l}$ of buffer containing $50 \mathrm{mM}$ Hepes, $150 \mathrm{mM} \mathrm{NaCl}, 10 \mathrm{mM} \mathrm{CaCl}$, and $10 \mathrm{mM}$ $\mathrm{MgCl}_{2}$ ( $\mathrm{pH} 7.5$ ), and incubated for $60 \mathrm{~min}$ at $37^{\circ} \mathrm{C}$. After incubation, unbound LDL particles were removed by washing the wells with $10 \mathrm{mM}$ Hepes buffer containing $50 \mathrm{mM} \mathrm{NaCl}, 2 \mathrm{mM} \mathrm{CaCl}_{2}$, and $2 \mathrm{mM} \mathrm{MgCl}_{2}$, and the amounts of bound LDL particles in the wells were determined using the Amplex Red Cholesterol assay kit (Invitrogen, Thermo Fisher Scientific) according to the manufacturer's instructions. Specific binding to PGs was calculated by subtracting the amount of LDL bound to the non-coated wells from the amount of LDL bound to the PG-coated wells, and the results are expressed as fold change relative to native LDL.

\subsection{Ex vivo binding of LDL to human carotid atherosclerotic lesions}

Frozen tissue sections $(10 \mu \mathrm{m})$ were cut from OCT (Tissue-Tek O.C.T. compound, Sakura) embedded carotid artery and stored at $-20^{\circ} \mathrm{C}$ until used. Human LDL, both atto 594-labeled LDL and non-labeled, was dialyzed against sample buffer [ $50 \mathrm{mM}$ Hepes buffer containing $30 \mathrm{mM} \mathrm{NaCl}, 2 \mathrm{mM} \mathrm{MgCl} 2$ and $2 \mathrm{mM} \mathrm{CaCl}_{2}(\mathrm{pH}$ $7.5)$ ], after which the LDL samples $(1 \mathrm{mg} / \mathrm{ml}$ ) were incubated in the presence or absence of $4.5 \mu \mathrm{g} / \mathrm{ml}$ cathepsin G overnight at $37^{\circ} \mathrm{C}$. OCT from the frozen tissue sections was removed by incubating the slides in DPBS for $20 \mathrm{~min}$ at room temperature, after which the slides were quickly rinsed with $\mathrm{H}_{2} \mathrm{O}$. In some cases, the tissue sections were pre-incubated for $60 \mathrm{~min}$ at $37^{\circ} \mathrm{C}$ with $50 \mathrm{mU}$ chondroitinase ABC (Amsbio, former Seikagaku) in $50 \mathrm{mM}$ Tris buffer containing $50 \mathrm{mM}$ sodium acetate and $0.01 \% \mathrm{BSA}(\mathrm{pH} 8.0)$. The slides were then washed twice with sample buffer and incubated with the LDL samples $(0.3 \mathrm{mg} / \mathrm{ml}$ in sample buffer) for $90 \mathrm{~min}$ at $37^{\circ} \mathrm{C}$ in a humidified chamber. The tissue sections were washed three times with sample buffer, quickly rinsed with $\mathrm{H}_{2} \mathrm{O}$, and finally mounted with Vectashield fluorescent mounting media with DAPI (Vector Laboratories). The images were acquired using Leica DM6000 microscope equipped with DFC420 camera and LAS AF (Leica Application Suite Advanced Fluorescence) software (Leica Microsystems).The differences in PG-binding between native atto 594-labeled LDL and cathepsin G-treated atto 594-labeled LDL were judged based on fluorescence intensities quantified using Image J software (NIH). Unlabeled LDL, both native and cathepsin G-treated, was used as negative control.

\subsection{Circular dichroism spectroscopy}

After incubation overnight with chymase, cathepsin G or MC releasate, samples of LDL $(50 \mu \mathrm{g} / \mathrm{ml})$ were analyzed by circular dichroism (CD) as described previously [33]. Before analyses, the neutral proteases were separated from LDL by extensive centrifugation (40 $000 \mathrm{rpm}, 2 \mathrm{~h}$ at $4{ }^{\circ} \mathrm{C}$ ).

\subsection{Electron microscopy}

LDL particles were negatively stained using 2\% neutral uranyl acetate, inspected under a Jeol JEM-1400 transmission electron microscope (Jeol Ltd., Tokyo, Japan) and photographed with Gatan Orius SC 1000B camera (Gatan Inc., Pleasanton, CA, USA) at the
Electron Microscopy Unit, Institute of Biotechnology, University of Helsinki. The diameters of 100-250 randomly selected lipoprotein particles were measured with Image Pro Plus software, version 6.2 (Media Cybernetics, Inc., Rockville, MD, USA) from the electron micrographs.

\subsection{Coronary and carotid samples}

Human coronary artery specimens for immunohistochemistry and quantitative PCR were selected from Wihuri Research Institute coronary specimen collection [34,35]. Samples were either from patients that underwent heart transplant surgery $(n=1$ for immunohistochemistry and $\mathrm{n}=4$ for RNA isolation, collected after informed consent of the patients) or from heart transplant donors ( $\mathrm{n}=5$ for RNA isolation) collected for research purposes in cases in which the candidate heart transplant did not fulfil the criteria for clinical use. The coronary tissue pieces had been snap-frozen in liquid nitrogen and stored at $-80^{\circ} \mathrm{C}$ (RNA isolation) or fixed (immunohistochemistry) immediately after the excision. Human carotid atherosclerotic plaques were obtained from patients undergoing carotid endarterectomy operation in the Department of Vascular Surgery in Helsinki University Hospital after informed consent from the patients. The carotid tissue pieces had been snapfrozen in liquid nitrogen and stored at $-80^{\circ} \mathrm{C}$ until further analysis. The use of human material conforms to the principles outlined in the Declaration of Helsinki and the study protocol was approved by the responsible Ethics Committees of the Helsinki University Hospital or the Finnish National Authority for Medicolegal Affairs.

\subsection{Immunohistochemistry of human coronary atherosclerotic plaque}

For immunohistochemical studies the coronary segments were treated and double immunostained against cathepsin $G$ and tryptase, as described previously [34], except that cathepsin $G$ was visualized with goat anti-rabbit IgG Alexa 488 (1:200, green) and tryptase with goat anti-mouse IgG1Alexa 546 (1:200, red, Molecular Probes). The numbers of tryptase-positive and cathepsin Gpositive cells were counted using Image J software (NIH).

\subsection{RNA isolation from human coronary arteries and quantitative $P C R$}

RNA was isolated from snap-frozen coronaries as previously described [35]. cDNA was synthesized from coronary RNA with High-Capacity cDNA Reverse Transcription Kit according to instructions of the manufacturer (Applied Biosystems). The cDNA was amplified in duplicates and the data were calculated as previously described [21] using the following gene-specific primers and probes: cathepsin G (CTSG) 5'-GGTTCCTGGTGCGAGAAGAC-3' (sense), 5'-GATGTGTTGCTGGGTGTTTTC-3' (anti-sense), and 5'AAGCAATATAAATGTCACCCTGGGCGC-3' (probe); chymase (CMA1) 5'-TGCTCATTGTGCAGGAAGGTC-3' (sense) and 5' ACCTCAAGCTTCTGCCATGTG-3' (anti-sense); tryptase (TPSAB1/ TPSB2) 5'-CGATGTGGACAATGATGAGC-3' (sense) and 5' CGCCAAGGTGGTATTTTGC-3' (anti-sense); CD68 5'-AGCTTTGGATTCATGCAGGAC-3' (sense) and 5'-ATCTCGAAGGGATGCATTCTG-3' (anti-sense); and proteinase 3 (PRTN3) 5'-TGTCACCGTGGTCACCTTCTT-3' (sense), 5'-CCCCAGATCACGAAGGAGTCTAT-3' (antisense), and 5'-TTGCACTTTCGTCCCTCGCCG-3' (probe). Target gene expression was normalized to glyceraldehyde-3-phosphate dehydrogenase (GAPDH) [5'-CCACATCGCTCAGACACCAT-3' (sense), 5' GGCAACAATATCCACTTTACCAGAG-3' (anti-sense), and 5'-CCAATACGACCAAATCCGTTGACTCC-3' (probe)]. 


\subsection{Statistical analysis}

Statistical analyses were performed with the GraphPad Prism software, version 6 (GraphPad Software, Inc., La Jolla, CA, USA) and SPSS for Windows software, version 20 (SPSS Inc., Chicago, Illinois, USA) using the nonparametric Kruskall-Wallis test with Dunn's multiple comparisons correction to compare the groups. $p<0.05$ was considered statistically significant. Correlations were investigated by calculation of Pearson's coefficient of correlation. The data in the figures are expressed as means \pm SEM.

\section{Results}

\subsection{Activated mast cells release a variety of neutral proteases}

At week 9 of culture, human MCs were activated with calcium ionophore to degranulate, and thereby to release their neutral granule proteases into the incubation medium. The amounts of the released enzymatically active proteases were then measured by activity assays using protease-specific substrates and/or inhibitors. The conditioned media, i.e. MC releasates contained $11.2 \pm 5.8 \mu \mathrm{g} /$ $\mathrm{ml}$ of tryptase, $1.3 \pm 0.4 \mu \mathrm{g} / \mathrm{ml}$ of chymase, $3.2 \pm 1.2 \mu \mathrm{g} / \mathrm{ml}$ of cathepsin G, and $0.09 \pm 0.1 \mu \mathrm{g} / \mathrm{ml}$ of granzyme $\mathrm{B}(\mathrm{n}=5$ donors in duplicate experiments).

\subsection{Cathepsin $G$ is the major apoB-100-degrading neutral protease of cultured human mast cells}

LDL was first incubated overnight with each of the neutral proteases present in MC releasate, after which the degree of proteolysis of apoB-100 was determined by TCA-precipitation, apoB100 ELISA, and SDS-PAGE. Treatment of LDL with the proteases resulted in proteolysis of apoB-100 at various degrees, as indicated by the release of small TCA-soluble apoB-100-derived peptides from the protease-treated LDL particles (Fig. 1A). The percentages of TCA-soluble fragments produced after treatment of LDL with the various proteases at concentrations found in the MC releasates revealed that cathepsin $\mathrm{G}$ led to most extensive proteolysis of apoB$100(12.9 \pm 0.9 \%)$, followed by chymase $(4.2 \pm 0.1 \%)$, tryptase $(3.3 \pm 0.1 \%)$, and CPA $(2.5 \pm 0.3 \%)$. Of note, granzyme B was practically unable to fragment apoB-100 into small TCA-soluble peptides. $\mathrm{MC}$ releasate containing all the above mentioned neutral proteases resulted in the generation of $6.8 \pm 0.5 \%$ TCA-soluble fragments. However, when all proteases, except with cathepsin $G$, had been inhibited with specific inhibitors, MC releasate resulted in more extensive proteolysis (about $10.7 \pm 2.3 \%$ TCA-soluble fragments), a finding in line with the results obtained with purified cathepsin G. Of note, the peptidic inhibitor, RETF- $(\mathrm{OPh})_{2}$, specifically inhibits chymase, while allowing full activity of cathepsin G [36], and was thus used to selectively inhibit chymase in MC releasates. ApoB ELISA, that specifically detected apoB- 100 but not apoB-48, indicated that, depending on the neutral protease used, treatment of LDL with MC neutral proteases induced loss of C-terminal immunoreactivity of apoB-100 at various degrees (Fig. 1B). Importantly, incubation of LDL with cathepsin G resulted in almost total loss of the apoB-100 C-terminal immunoreactivity.

The cleavage patterns of apoB- 100 by MC releasate, tryptase, chymase, and cathepsin G were visualized with SDS-PAGE. As shown in Fig. $1 C$ and D, incubation of LDL with human MC releasate led to extensive degradation of apoB-100. Comparison of the apoB bands formed after incubation of LDL with tryptase, chymase, or cathepsin $\mathrm{G}$ showed that each neutral protease generated a specific cleavage pattern of apoB-100. CPA3 and granzyme B were excluded from this experiment because CPA3, as an exopeptidase only cleaves the C-terminal amino acid, and granzyme $\mathrm{B}$, again, appeared not to degrade apoB-100, when examined with the TCAassay and apoB-100 ELISA, as described above. The presence of PGs in the LDL incubation mixtures clearly affected the cleavage pattern of apoB-100 generated by cathepsin G, whereas their presence appeared not to have a significant effect on the chymase-dependent cleavage pattern of apoB-100 (Fig. 1D). In fact, the cleavage pattern of apoB-100 treated by cathepsin $\mathrm{G}$ in the presence PGs resembled that generated by $\mathrm{MC}$ releasate.

\subsection{Cathepsin G-and mast cell releasate-dependent proteolysis of LDL increases its binding to proteoglycans}

PG-binding of native and modified LDL was determined by incubation of the LDL samples with isolated human aortic PGs, and measuring the amount of cholesterol of the bound LDL with Amplex Red cholesterol assay. Treatment of LDL with MC releasate increased the binding of LDL to human aortic PGs by almost 4-fold compared with native LDL (Fig. 2A). Similar fold increase in PGbinding was also observed when LDL was treated with cathepsin $G$, whereas treatment of LDL with chymase increased the PGbinding by 2 -fold. In contrast, treatment of LDL with tryptase generated LDL particles with significantly decreased PG-binding, when compared with untreated LDL particles. The binding of LDL to human atherosclerotic plaques was evaluated by incubating fluorescently labeled LDL, both native and cathepsin G-treated with frozen tissue sections of human carotid plaques. We found that treatment of LDL with cathepsin G increased remarkably the binding of LDL to the plaques, as indicated by differences in the fluorescence intensities (Fig. 2B and C). Furthermore, enzymatic digestion of chondroitin sulfate caused a drastic reduction in the binding of the cathepsin G-treated LDL (Fig. 2C and D), indicating that chondroitin sulfates of the extracellular matrix play an important role in mediating the binding of LDL to the atherosclerotic plaques. Unlabeled LDL used as a negative control is shown in Fig. 2E.

\subsection{Cathepsin G-dependent proteolysis of $L D L$ induces conformational changes in apoB-100}

To study the effect of LDL proteolysis on secondary structure of apoB-100, conformational changes of apoB- 100 was analyzed by circular dichroism. To exclude the contribution of $\mathrm{CD}$ of the neutral proteases per se, LDL particles were first flotated by centrifugation at $40000 \mathrm{rpm}$, for $2 \mathrm{~h}$, at $4{ }^{\circ} \mathrm{C}$, to separate the neutral proteases from LDL. Compared to control LDL particles (Fig. 3A) the spectra of proteolyzed LDL have similar shape but show a slight decrease in CD intensity, indicating that proteolysis of LDL by MC proteases, especially with cathepsin $G$ resulted in conformational changes of apoB-100. The difference between the CD spectra of native LDL and of cathepsin G-treated LDL is shown in Fig. 3B.

\subsection{Cathepsin $G$ and mast cell releasate trigger fusion of $L D L$ particles}

Proteolysis of apoB-100 has been shown to promote fusion of LDL particles resulting in formation of enlarged particles $[9,30]$. The effect of proteolysis on LDL particle fusion was examined using transmission electron microscopy to produce electron micrographs from which the diameters of negatively stained differentially treated LDL particles were measured (Fig. 4A and B). The diameter of the native LDL particles was $22.0 \pm 0.40 \mathrm{~nm}$. However, treatment of LDL with MC releasate, cathepsin G, or chymase led to the formation of enlarged (i.e., fused) LDL particles and to a more skewed size distribution. Indeed, the diameters of LDL particles were $29.5 \pm 0.70 \mathrm{~nm}, 30.3 \pm 0.60 \mathrm{~nm}$, and $27.0 \pm 0.32 \mathrm{~nm}$ when LDL were 
A

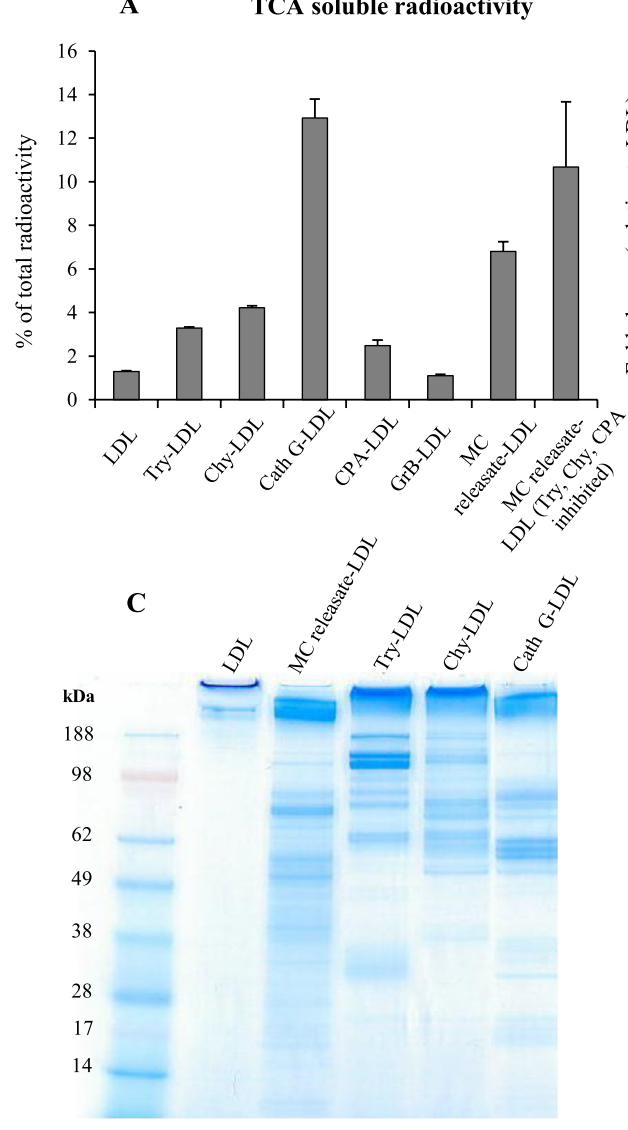

B
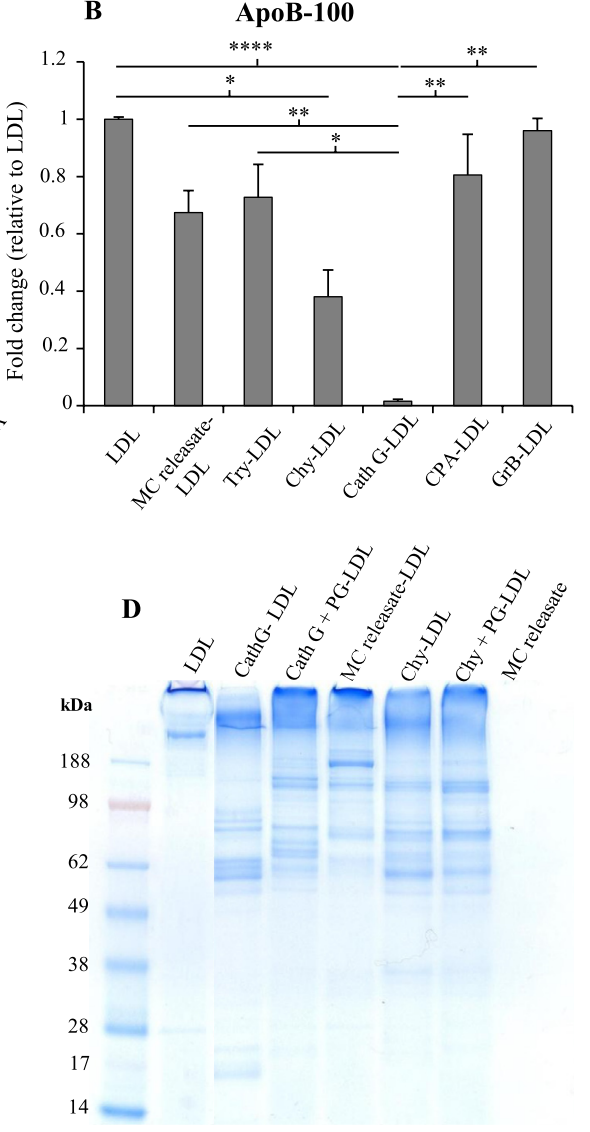

Fig. 1. Proteolysis of apoB-100 of LDL by mast cell neutral proteases.

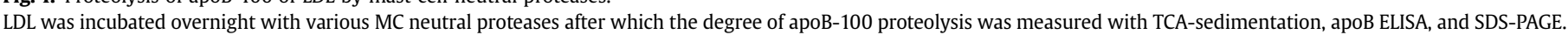

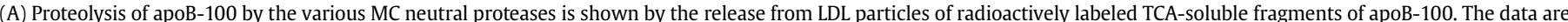

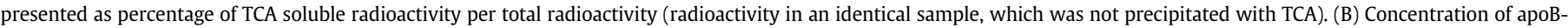

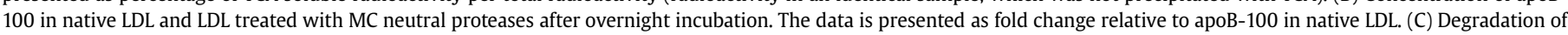

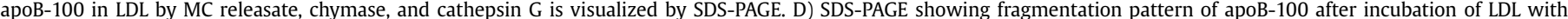

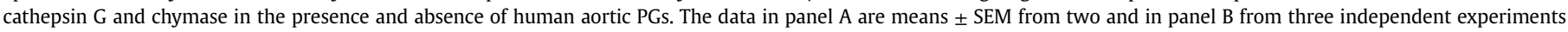

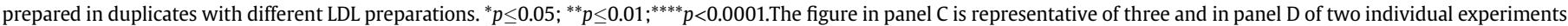

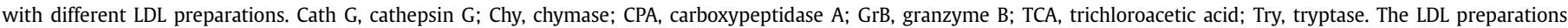
proteolyzed individually with the above-listed proteases are labeled using the above abbreviations as prefixes.

treated with MC releasate, cathepsin G, and chymase, respectively. Fig. 4C shows the approximate diameters of particles generated by fusion of 2-100 LDL particles, and based on this, the average-sized fusion particle resulted from fusion of 2-3 LDL proteolyzed LDL particles. The largest particles observed by electron microscopy had diameters of about $100 \mathrm{~nm}$ and based on the calculations, they had been generated when about 100 of the normal-sized 22-nm LDL particles had fused to form a single particle.

\subsection{Mast cells are the major cathepsin G-positive type of cell in atherosclerotic coronary plaques}

To identify the presence of MCs in atherosclerotic lesions, sections of human coronary arterial plaque with histomorphological characteristics of a stable plaque were immunostained against cathepsin $\mathrm{G}$ and tryptase, the latter one indicating the presence of MC. Representative double immunofluorescence staining of cathepsin $G$ and tryptase in a coronary plaque is presented in Fig. 5A-D and shows the presence of cathepsin G-positive MCs in the plaque. In fact, of all cathepsin G-positive cells 69\% were MCs, i.e. cathepsin G-positive tryptase-positive cells, a finding which is in accordance with a previous study of our laboratory reporting that
MCs represent, on average, 73\% of all cathepsin G-positive cells in human coronary plaques [34]. The plaque also contained some cathepsin G-positive cells which were tryptase-negative, i.e., they were not MCs. The tryptase-negative cathepsin G-positive cells were not specified further.

\subsection{Cathepsin $G$ expression correlates with mast cell tryptase and} chymase but not with neutrophil proteinase 3 in coronary plaques

To further evaluate the candidate cell types expressing cathepsin $\mathrm{G}$ in atherosclerotic plaques, the expression of cathepsin $\mathrm{G}$, the MC-specific chymase and tryptase, neutrophil proteinase 3, and CD68, the latter of which is mainly expressed by monocytes and macrophages, but also by MCs, were analyzed both in coronary segments of AHA classes I-II and in advanced atherosclerotic plaques (AHA classes IV-V) from 9 patients [35]. We found a significant correlation between the expression of lesional cathepsin $\mathrm{G}$ and the MC chymase and tryptase, and to a lesser extent between lesional cathepsin $\mathrm{G}$ and $\mathrm{CD} 68$, but not between cathepsin $\mathrm{G}$ and neutrophil proteinase 3 (Fig. 5E-H). The data are presented as fold changes relative to normal coronary segment of each patient. 

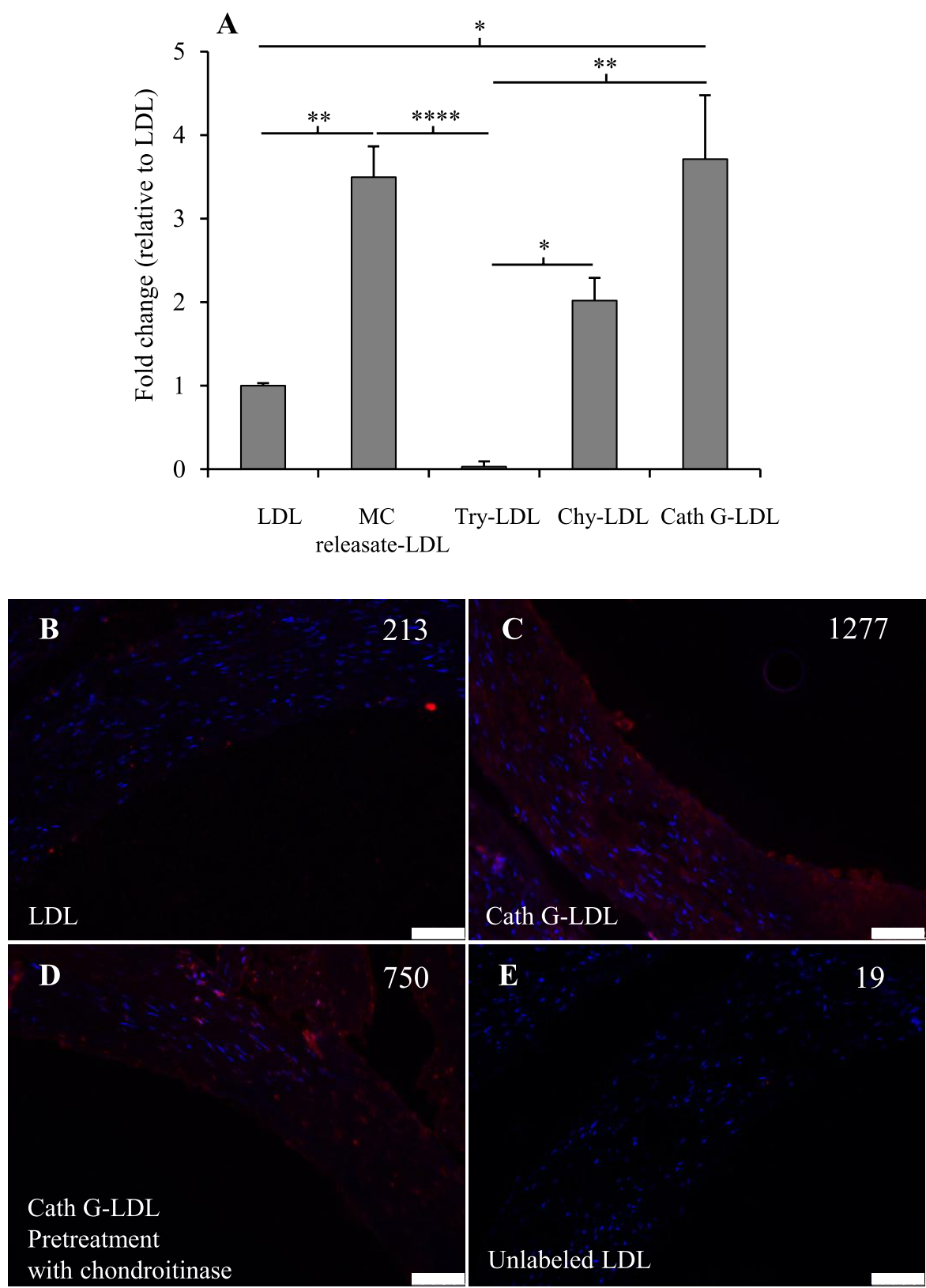

Fig. 2. Binding of modified LDL to human aortic proteoglycans and to human carotid atherosclerotic lesions.

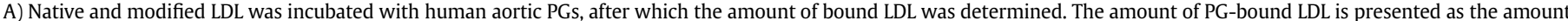

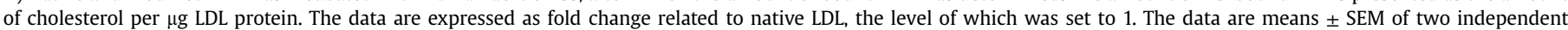

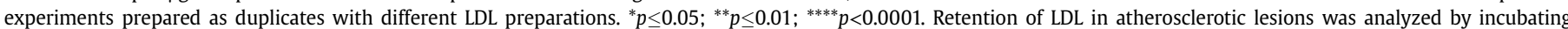

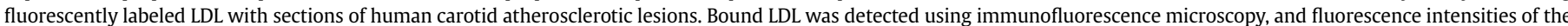

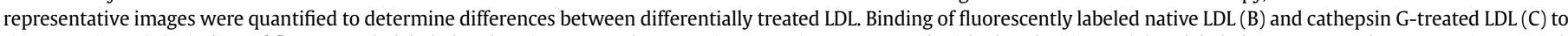

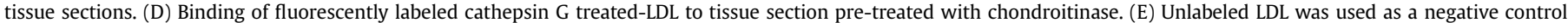
Fluorescence intensities are shown in the upper right hand corner. Scale bars $=100 \mu \mathrm{m}$.

\section{Discussion}

In the present study, we investigated the proteolytic activity towards apoB-100 of LDL of various neutral proteases known to be present in the cytoplasmic secretory granules of human MCs, namely tryptase, chymase, CPA3, cathepsin G, and granzyme B. The present data demonstrate that cathepsin $G$ efficiently degrades apoB-100 of LDL, and that this enzyme is the major apoB-100degrading neutral granule protease in human MCs. Proteolysis of LDL with cathepsin G led to increase both in the size and in the binding of the proteolyzed LDL to isolated human aortic PGs and
PGs in human carotid atherosclerotic lesions ex vivo. Since atherogenicity of LDL is linked to its ability to bind PGs [1], novel mechanisms leading to increased PG binding of LDL are likely to contribute to better understanding of the molecular bases behind the pathogenesis of this disease.

Cathepsin G is a serine protease expressed in MCs and neutrophils [19]. In human MCs, cathepsin G is the closest relative of chymase and it shares some functional properties with human chymase, such as the ability to convert angiotensin II from angiotensin I [37]. Similar to chymase, cathepsin G has a chymotrypsinlike activity, that is, a preference of cleaving peptide and protein 

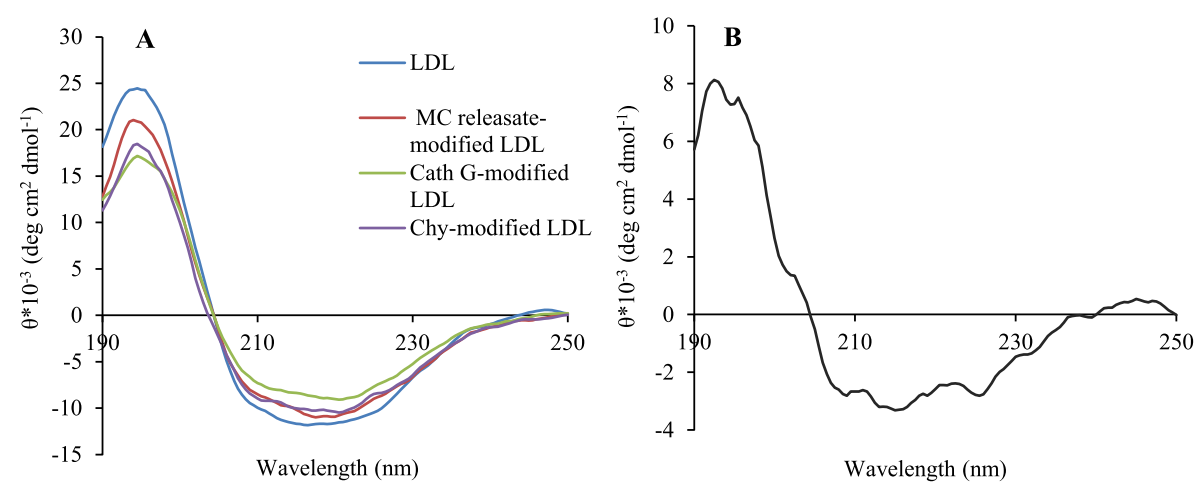

Fig. 3. Conformational changes in the secondary structure of apoB-100 upon proteolysis of LDL.

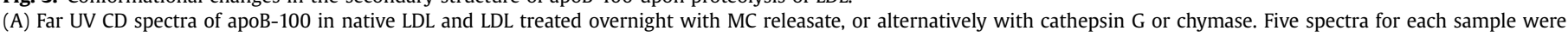

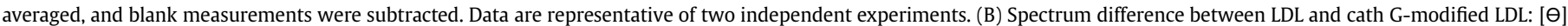
LDL - $[\Theta]$ cath G-modified LDL. UV, ultraviolet; CD, circular dichroism.
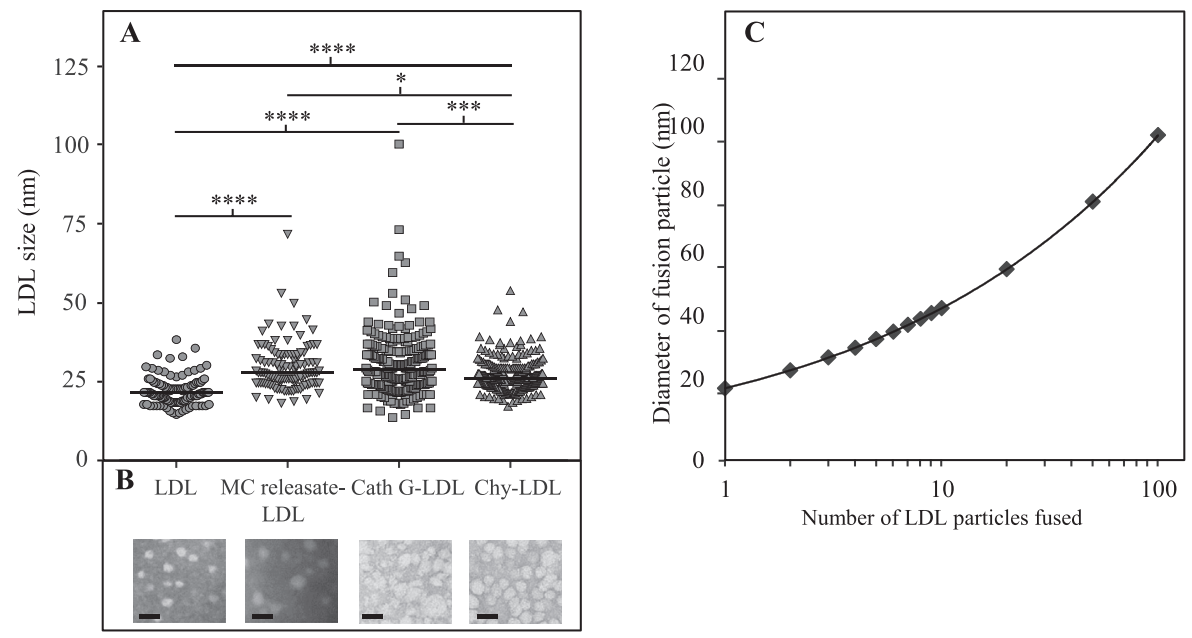

Fig. 4. Size distribution of differentially modified LDL particles.

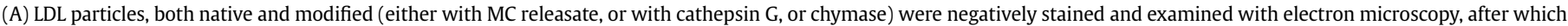

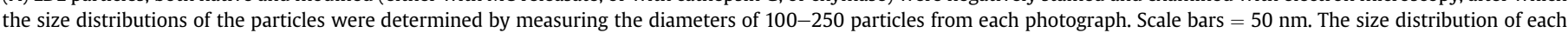

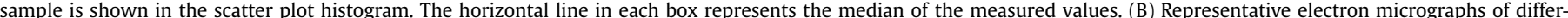

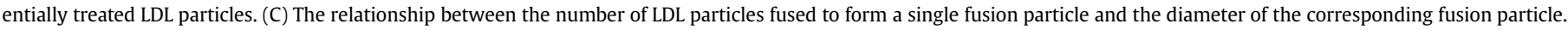
${ }^{*} p \leq 0.05 ;{ }^{* * *} p \leq 0.001 ;{ }^{* * * *} p<0.0001$.

substrates at the C-terminal site of aromatic amino acids, such as phenylalanine, tyrosine, and tryptophan. However, compared to chymase it has a broader cleavage specificity by possessing, in addition to chymotrypsin-like activity, also trypsin-like activity [38]. This is likely to explain the more potent destructive potential of cathepsin G compared with chymase towards apoB-100.

Studies of human subjects have provided evidence that cathepsin $G$ expression in the arterial wall increases during atherogenesis. Thus, when compared with normal human aortas, the expression of cathepsin $\mathrm{G}$ and chymase in atherosclerotic aortas was found to be increased [39]. In line with the above observation, human atherosclerotic lesions have been shown to contain significantly higher numbers of cathepsin G-positive MCs compared to control vessels [40]. In a previous immunohistochemical study [34], we compared the proportions of two cathepsin G-containing cells, MCs and neutrophils, in atherosclerotic lesion of human coronary artery, and found a MC-to-neutrophil ratio of 5: 1. Although cathepsin $\mathrm{G}$ has been mainly associated with these two cell types, in human carotid atherosclerotic lesions also macrophages, smooth muscle cells, CD31-positive endothelial cells, and CD4 T cells appear to contain cathepsin G [41]. Yet, in accordance with the previous finding [34], we now found that in human coronary atherosclerotic lesions most cathepsin G-containing cells also contained the MCspecific neutral protease tryptase and that the expression of cathepsin $\mathrm{G}$ correlates remarkably with the expression of chymase and tryptase, but not with neutrophil proteinase 3 , findings indicating that MCs are a major local source of cathepsin $G$ in human atherosclerotic lesions, at least in the stable phase of the disease.

Interestingly, cathepsin $\mathrm{G}$ activity in the circulating blood has an LDL-lowering effect, which was found to be associated with reduced atherosclerosis in experimental animals, and was suggested to possess a protective effect against atherosclerosis also in humans [41]. It is worth noting that MCs are found in circulation only as agranular progenitor cells and accumulate cathepsin $\mathrm{G}$ and other neutral granule proteases only during differentiation in peripheral tissues [21]. Thus in the bloodstream, neutrophils appear to represent the major cathepsin G-containing cell type. In the circulating blood, cathepsin $\mathrm{G}$ on neutrophil surface remains active, and may so degrade the apoB-100 component of LDL, thereby modifying the particles to be cleared more rapidly by, e.g. the liver, 

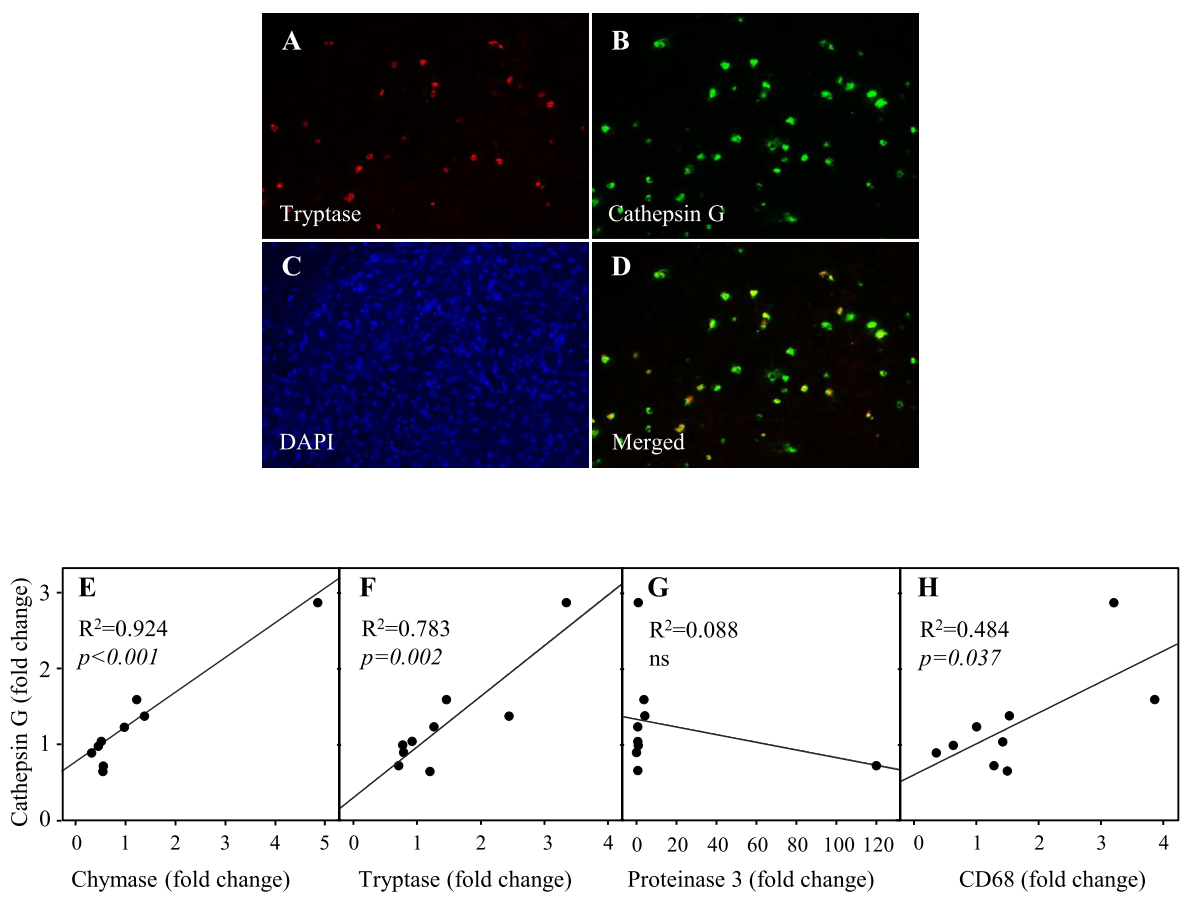

Fig. 5. Expression of cathepsin G, tryptase, chymase, proteinase 3, and CD68 in human coronary artery specimens.

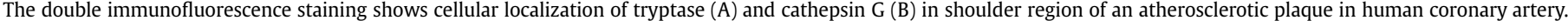

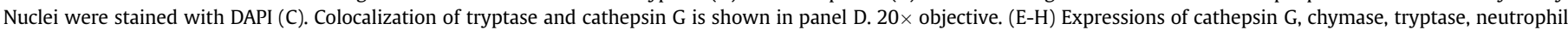

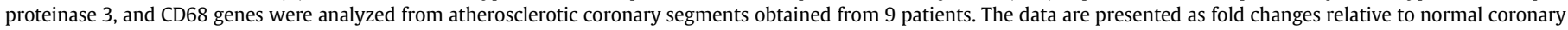
segment of each patient. Correlation of the expression of cathepsin $G$ with that of (E) chymase, (F) tryptase, (G) proteinase 3, and (H) CD68 are shown.

and so reducing exposure of the arterial wall to circulating LDL particles. In sharp contrast, the apoB-100-degrading activity of cathepsin $G$ in the arterial intima appears to be solely proatherogenic, and based on the previous [34] and the present data, highly attributed to MCs.

In the context of cardiovascular diseases, cathepsin $G$ is best known for its angiotensin-converting activity [37]. In addition, cathepsin $G$ activates matrix metalloproteinases [42-44] that have been shown to participate in atherogenesis [45], and may degrade VE-cadherin [34], an important structural component for the integrity of the arterial endothelium. Cathepsin $\mathrm{G}$ is also involved in monocyte and neutrophil chemotaxis [46]. Interestingly, studies using genetically modified $\mathrm{Apoe}^{-I-}$ and $\mathrm{CatG}^{-/-}$mice showed that cathepsin $\mathrm{G}$ controls arterial, but not venous, myeloid cell recruitment, and lack of cathepsin $G$ protected the mice from atherosclerosis [47]. By showing that cathepsin $G$ efficiently degrades apoB-100 of LDL with ensuing increase in PG-binding of the proteolyzed LDL, the present study adds a novel mechanism by which cathepsin $\mathrm{G}$ may contribute to atherogenesis.

Initially it was found that secretory granules exocytosed from rat MCs degradade apoB-100 in LDL particles [48]. Subsequent studies showed that heparin present in the exocytosed rat MC granules is capable of binding apoB-100 of LDL [49], thus facilitating proteolysis of apoB-100 by the, also granule-bound, chymase and CPA [50]. The sequential proteolysis of apoB- 100 by the two neutral proteases resulted in fusion of LDL particles with ensuing formation of larger lipoprotein particles on MC granules [51]. Importantly, both proteolysis and fusion of the rat granule -treated LDL particles were found to contribute to increased binding of LDL to the heparin glycosaminoglycan chains of the granule PGs [52]. It is likely that proteolysis of apoB- 100 on the surface of the modified LDL particles induces conformational changes in it, thereby increasing its ability to interact with PGs. In the present study, proteolysis of LDL with cathepsin $G$ was associated with conformational changes in the secondary structure of apoB-100, which may have exposed cryptic domains for PG binding [9], such as the residues 3148-3158 (referred to as site A) that becomes exposed in phospholipase A2modified LDL resulting in increased PG-binding of the phospholipase A2-treated LDL [10]. In the present study, tryptase treatment nearly completely abolished the binding of LDL to PGs. A reasonable explanation for this observation is that tryptase-induced fragmentation of apoB-100 leads to conformational changes in it, and that they block the main PG-binding site of apoB-100.

Proteolysis-induced fusion of LDL requires release of apoBfragments from LDL particles [30]. ApoB-100 covers about 1/3 of LDL surface and proteolytic removal of parts of this protein is thought to lead to loosening of the surface lipid monolayer of the modified particles followed by their destabilization and fusion [7,53]. In the present study, we show that proteolysis of LDL with cathepsin G or chymase or with MC releasate leads to release of apoB-fragments from the modified LDL and increases the size of LDL particles from approximately $22 \mathrm{~nm}$ to $30 \mathrm{~nm}$, thus resulting in the formation of enlarged LDL particles that fall within the range of the extracellular lipid particles in the human atherosclerotic arterial intima [11,54]. Particle fusion may induce an increase in PGbinding strength due to an increase in the total number of exposed positively charged domains, i.e., of potential PG-binding sites, on the surface of modified LDL, as also previously suggested [9]. Indeed, as proteolytic fusion of LDL leads to accumulation of non-releasable apoB-100 fragments of several proteolyzed LDL particles in a single fused LDL particle, formation of large particles with multiple copies of PG-binding sites may ensue [9]. Since cathepsin G-, chymase-, and MC releasate-modified LDL particles showed signs of fusion, the above-described mechanism may partially explain the increased PG-binding of these particles. Taken together, proteolytic fusion provides a plausible mechanism by 
which cathepsin $G$ and chymase released by activated mast cells induce degradation of apoB-100 and increase the PG-binding of the modified LDL, yielding LDL particles with potent atherogenic properties.

\section{Conflicts of interest}

The authors declare that they do not have anything to disclose regarding conflict of interest with respect to this manuscript.

\section{Author contribution}

K.M. participated in the study design and conducted the experiments with the exception of double immunofluorescence stainings of coronary arteries, which were conducted by M.I.M. S.D.N. performed the CD analysis, K.R. isolated RNA from coronary artery samples, and R.P. and K.Ö. performed electron microscopy of the LDL particles. P.J.L. provided human carotid endarterectomy specimens and offered expert advice. K.M. conducted all the data analyses, interpreted the results, and wrote the manuscript. P.T.K. and K.Ö participated in the writing. S.D.N., M.I.M., R.P, P.T.K., and K.Ö. participated in the study design, data analyses, interpretation of the results, and editing the manuscript.

\section{Acknowledgements}

We thank Maija Atuegwu and Mari Jokinen for excellent technical assistance. The Wihuri Research Institute is maintained by Jenny and Antti Wihuri Foundation. This study was also supported by Paavo Nurmi Foundation (K.M.), Ida Montin Foundation (K.M.), Meilahti Foundation (K.M.), Finnish Foundation for Cardiovascular Research (K.M. and K.Ö.), Paulo Foundation (K.R.), and the Academy of Finland (grant number 265940 to K.Ö.).

\section{References}

[1] J. Boren, K.J. Williams, The central role of arterial retention of cholesterol-rich apolipoprotein-B-containing lipoproteins in the pathogenesis of atherosclerosis: a triumph of simplicity, Curr. Opin. Lipidol. 27 (2016) 473-483.

[2] J. Boren, K. Olin, I. Lee, A. Chait, T.N. Wight, T.L. Innerarity, Identification of the principal proteoglycan-binding site in LDL. A single-point mutation in apoB100 severely affects proteoglycan interaction without affecting LDL receptor binding, J. Clin. Invest. 101 (1998) 2658-2664.

[3] K. Skålen, M. Gustafsson, E.K. Rydberg, L.M. Hulten, O. Wiklund, T.L. Innerarity, J. Boren, Subendothelial retention of atherogenic lipoproteins in early atherosclerosis, Nature 417 (2002) 750-754.

[4] G. Camejo, B. Rosengren, U. Olson, F. Lopez, S.O. Olofson, C. Westerlund, G. Bondjers, Molecular basis of the association of arterial proteoglycans with low density lipoproteins: its effect on the structure of the lipoprotein particle Eur. Heart J. 11 (Suppl E) (1990) 164-173.

[5] G. Camejo, E. Hurt-Camejo, O. Wiklund, G. Bondjers, Association of apo B lipoproteins with arterial proteoglycans: pathological significance and molecular basis, Atherosclerosis 139 (1998) 205-222.

[6] R. Plihtari, E. Hurt-Camejo, K. Öörni, P.T. Kovanen, Proteolysis sensitizes LDL particles to phospholipolysis by secretory phospholipase A2 group V and secretory sphingomyelinase, J. Lipid Res. 51 (2010) 1801-1809.

[7] K. Öörni, M.O. Pentikäinen, M. Ala-Korpela, P.T. Kovanen, Aggregation, fusion, and vesicle formation of modified low density lipoprotein particles: molecular mechanisms and effects on matrix interactions, J. Lipid Res. 41 (2000) $1703-1714$

[8] J.K. Hakala, K. Öörni, M.O. Pentikäinen, E. Hurt-Camejo, P.T. Kovanen, Lipolysis of LDL by human secretory phospholipase $\mathrm{A}(2)$ induces particle fusion and enhances the retention of LDL to human aortic proteoglycans, Arterioscler. Thromb. Vasc. Biol. 21 (2001) 1053-1058.

[9] K. Paananen, J. Saarinen, A. Annila, P.T. Kovanen, Proteolysis and fusion of low density lipoprotein particles strengthen their binding to human aortic proteoglycans, J. Biol. Chem. 270 (1995) 12257-12262.

[10] C. Flood, M. Gustafsson, R.E. Pitas, L. Arnaboldi, R.L. Walzem, J. Boren, Molecular mechanism for changes in proteoglycan binding on compositional changes of the core and the surface of low-density lipoprotein-containing human apolipoprotein B100, Arterioscler. Thromb. Vasc. Biol. 24 (2004) 564-570.

[11] S. Lehti, S.D. Nguyen, I. Belevich, H. Vihinen, H.M. Heikkilä, R. Soliymani, R. Käkelä, J. Saksi, M. Jauhiainen, G.A. Grabowski, O. Kummu, S. Hörkkö,
M. Baumann, P.J. Lindsberg, E. Jokitalo, P.T. Kovanen, K. Öörni, Extracellular lipids accumulate in human carotid arteries as distinct three-dimensional structures and have proinflammatory properties, Am. J. Pathol. 188 (2018) $525-538$.

[12] G.K. Sukhova, G.P. Shi, D.I. Simon, H.A. Chapman, P. Libby, Expression of the elastolytic cathepsins $\mathrm{S}$ and $\mathrm{K}$ in human atheroma and regulation of their production in smooth muscle cells, J. Clin. Invest. 102 (1998) 576-583.

[13] J.K. Hakala, R. Oksjoki, P. Laine, H. Du, G.A. Grabowski, P.T. Kovanen, M.O. Pentikäinen, Lysosomal enzymes are released from cultured human macrophages, hydrolyze LDL in vitro, and are present extracellularly in human atherosclerotic lesions, Arterioscler. Thromb. Vasc. Biol. 23 (2003) $1430-1436$

[14] K. Ö̈rni, M. Sneck, D. Bromme, M.O. Pentikäinen, K.A. Lindstedt, M. Mäyränpää, H. Aitio, P.T. Kovanen, Cysteine protease cathepsin F is expressed in human atherosclerotic lesions, is secreted by cultured macrophages, and modifies low density lipoprotein particles in vitro, J. Biol. Chem. 279 (2004) 34776-34784.

[15] M. Kaartinen, A. Penttilä, P.T. Kovanen, Mast cells of two types differing in neutral protease composition in the human aortic intima. Demonstration of tryptase- and tryptase/chymase-containing mast cells in normal intimas, fatty streaks, and the shoulder region of atheromas, Arterioscler. Thromb. 14 (1994) 966-972.

[16] M. Kaartinen, A. Penttilä, P.T. Kovanen, Accumulation of activated mast cells in the shoulder region of human coronary atheroma, the predilection site of atheromatous rupture, Circulation 90 (1994) 1669-1678.

[17] M. Jeziorska, C. McCollum, D.E. Woolley, Mast cell distribution, activation, and phenotype in atherosclerotic lesions of human carotid arteries, J. Pathol. 182 (1997) 115-122.

[18] L.B. Schwartz, A.M. Irani, K. Roller, M.C. Castells, N.M. Schechter, Quantitation of histamine, tryptase, and chymase in dispersed human T and TC mast cells, J. Immunol. 138 (1987) 2611-2615.

[19] G.H. Caughey, Mast cell proteases as pharmacological targets, Eur. J. Pharmacol. 778 (2016) 44-55.

[20] M.C. Strik, PJ. de Koning M.J. Kleijmeer, B.A. Bladergroen, A.M. Wolbink, J.M. Griffith, D. Wouters, Y. Fukuoka, L.B. Schwartz, C.E. Hack, S.M. van Ham, J.A. Kummer, Human mast cells produce and release the cytotoxic lymphocyte associated protease granzyme B upon activation, Mol. Immunol. 44 (2007) $3462-3472$.

[21] K. Maaninka, J. Lappalainen, P.T. Kovanen, Human mast cells arise from a common circulating progenitor, J. Allergy Clin. Immunol. 132 (2013), e463, 463-469.

[22] N.M. Schechter, A.M. Irani, J.L. Sprows, J. Abernethy, B. Wintroub, L.B. Schwartz, Identification of a cathepsin G-like proteinase in the MCTC type of human mast cell, J. Immunol. 145 (1990) 2652-2661.

[23] J. Lappalainen, K.A. Lindstedt, P.T. Kovanen, A protocol for generating high numbers of mature and functional human mast cells from peripheral blood, Clin. Exp. Allergy 37 (2007) 1404-1414.

[24] E. Hurt-Camejo, G. Camejo, B. Rosengren, F. Lopez, O. Wiklund, G. Bondjers, Differential uptake of proteoglycan-selected subfractions of low density lipoprotein by human macrophages, J. Lipid Res. 31 (1990) 1387-1398.

[25] K. Oörni, M.O. Pentikäinen, A. Annila, P.T. Kovanen, Oxidation of low density lipoprotein particles decreases their ability to bind to human aortic proteoglycans. Dependence on oxidative modification of the lysine residues, J. Biol. Chem. 272 (1997) 21303-21311.

[26] P.M. Bartold, R.C. Page, A microdetermination method for assaying glycosaminoglycans and proteoglycans, Anal. Biochem. 150 (1985) 320-324.

[27] R.J. Havel, H.A. Eder, J.H. Bragdon, The distribution and chemical composition of ultracentrifugally separated lipoproteins in human serum, J. Clin. Invest. 34 (1955) 1345-1353.

[28] C.M. Radding, D. Steinberg, Studies on the synthesis and secretion of serum lipoproteins by rat liver slices, J. Clin. Invest. 39 (1960) 1560-1569.

[29] A.E. Bolton, W.M. Hunter, The labelling of proteins to high specific radioactivities by conjugation to a 125I-containing acylating agent, Biochem. J. 133 (1973) 529-539.

[30] M. Piha, L. Lindstedt, P.T. Kovanen, Fusion of proteolyzed low-density lipoprotein in the fluid phase: a novel mechanism generating atherogenic lipoprotein particles, Biochemistry 34 (1995) 10120-10129.

[31] J.R. Hessler, D.W. Morel, L.J. Lewis, G.M. Chisolm, Lipoprotein oxidation and lipoprotein-induced cytotoxicity, Arteriosclerosis 3 (1983) 215-222.

[32] L.B. Steffensen, M.B. Mortensen, M. Kjolby, M.K. Hagensen, C. Oxvig, J.F. Bentzon, Disturbed laminar blood flow vastly augments lipoprotein retention in the artery wall: a key mechanism distinguishing susceptible from resistant sites, Arterioscler. Thromb. Vasc. Biol. 35 (2015) 1928-1935.

[33] S.D. Nguyen, K. Öörni, M. Lee-Rueckert, T. Pihlajamaa, J. Metso, M. Jauhiainen, P.T. Kovanen, Spontaneous remodeling of HDL particles at acidic pH enhances their capacity to induce cholesterol efflux from human macrophage foam cells, J. Lipid Res. 53 (2012) 2115-2125.

[34] M.I. Mäyränpää, H.M. Heikkilä, K.A. Lindstedt, A.F. Walls, P.T. Kovanen, Desquamation of human coronary artery endothelium by human mast cell proteases: implications for plaque erosion, Coron. Artery Dis. 17 (2006) $611-621$.

[35] K. Rajamäki, M.I. Mäyranpää, A. Risco, J. Tuimala, K. Nurmi, A. Cuenda, K.K. Eklund, K. Oörni, P.T. Kovanen, p38delta MAPK: a novel regulator of NLRP3 inflammasome activation with increased expression in coronary atherogenesis, Arterioscler. Thromb. Vasc. Biol. 36 (2016) 1937-1946. 
[36] W.W. Raymond, S.W. Ruggles, C.S. Craik, G.H. Caughey, Albumin is a substrate of human chymase. Prediction by combinatorial peptide screening and development of a selective inhibitor based on the albumin cleavage site, J. Biol. Chem. 278 (2003) 34517-34524.

[37] C.F. Reilly, D.A. Tewksbury, N.M. Schechter, J. Travis, Rapid conversion of angiotensin I to angiotensin II by neutrophil and mast cell proteinases, J. Biol. Chem. 257 (1982) 8619-8622.

[38] W.W. Raymond, N.N. Trivedi, A. Makarova, M. Ray, C.S. Craik, G.H. Caughey, How immune peptidases change specificity: cathepsin G gained tryptic function but lost efficiency during primate evolution, J. Immunol. 185 (2010) 5360-5368.

[39] E. Kaschina, H. Scholz, U.M. Steckelings, M. Sommerfeld, U.R. Kemnitz, M. Artuc, S. Schmidt, T. Unger, Transition from atherosclerosis to aortic aneurysm in humans coincides with an increased expression of RAS components, Atherosclerosis 205 (2009) 396-403.

[40] I. Rohm, S. Sattler, Y. Atiskova, D. Kretzschmar, R. Pistulli, M. Franz, C. Jung, G. Mall, T. Kronert, P.C. Schulze, A. Yilmaz, Increased number of mast cells in atherosclerotic lesions correlates with the presence of myeloid but not plasmacytoid dendritic cells as well as pro-inflammatory T cells, Clin. Lab. 62 (2016) 2293-2303.

[41] J. Wang, S. Sjöberg, T.T. Tang, K. Öörni, W. Wu, C. Liu, B. Secco, V. Tia, G.K. Sukhova, C. Fernandes, A. Lesner, P.T. Kovanen, P. Libby, X. Cheng, G.P. Shi, Cathepsin G activity lowers plasma LDL and reduces atherosclerosis, Biochim. Biophys. Acta 1842 (2014) 2174-2183.

[42] Y. Okada, I. Nakanishi, Activation of matrix metalloproteinase 3 (stromelysin) and matrix metalloproteinase 2 ('gelatinase') by human neutrophil elastase and cathepsin G, FEBS Lett. 249 (1989) 353-356.

[43] Y. Okada, Y. Gonoji, K. Naka, K. Tomita, I. Nakanishi, K. Iwata, K. Yamashita, T. Hayakawa, Matrix metalloproteinase 9 (92-kDa gelatinase/type IV collagenase) from HT 1080 human fibrosarcoma cells. Purification and activation of the precursor and enzymic properties, J. Biol. Chem. 267 (1992) 21712-21719.

[44] P. Shamamian, J.D. Schwartz, B.J. Pocock, S. Monea, D. Whiting, S.G. Marcus, P. Mignatti, Activation of progelatinase A (MMP-2) by neutrophil elastase, cathepsin G, and proteinase-3: a role for inflammatory cells in tumor invasion and angiogenesis, J. Cell. Physiol. 189 (2001) 197-206.
[45] B.A. Brown, H. Williams, S.J. George, Evidence for the involvement of matrixdegrading metalloproteinases (MMPs) in atherosclerosis, Prog. Mol. Biol Transl. Sci. 147 (2017) 197-237.

[46] O. Chertov, H. Ueda, L.L. Xu, K. Tani, W.J. Murphy, J.M. Wang, O.M. Howard T.J. Sayers, J.J. Oppenheim, Identification of human neutrophil-derived cathepsin G and azurocidin/CAP37 as chemoattractants for mononuclear cells and neutrophils, J. Exp. Med. 186 (1997) 739-747.

[47] A. Ortega-Gomez, M. Salvermoser, J. Rossaint, R. Pick, J. Brauner, P. Lemnitzer, J. Tilgner, R.J. de Jong, R.T. Megens, J. Jamasbi, Y. Doring, C.T. Pham, C. Scheiermann, W. Siess, M. Drechsler, C. Weber, J. Grommes, A. Zarbock, B. Walzog, O. Soehnlein, Cathepsin G controls arterial but not venular myeloid cell recruitment, Circulation 134 (2016) 1176-1188.

[48] J.O. Kokkonen, P.T. Kovanen, Low density lipoprotein degradation by rat mast cells. Demonstration of extracellular proteolysis caused by mast cell granules, J. Biol. Chem. 260 (1985) 14756-14763.

[49] J.O. Kokkonen, P.T. Kovanen, Low-density-lipoprotein binding by mast-cell granules. Demonstration of binding of apolipoprotein B to heparin proteoglycan of exocytosed granules, Biochem. J. 241 (1987) 583-589.

[50] J.O. Kokkonen, M. Vartiainen, P.T. Kovanen, Low density lipoprotein degradation by secretory granules of rat mast cells. Sequential degradation of apolipoprotein B by granule chymase and carboxypeptidase A, J. Biol. Chem. 261 (1986) 16067-16072.

[51] J.O. Kokkonen, P.T. Kovanen, Proteolytic enzymes of mast cell granules degrade low density lipoproteins and promote their granule-mediated uptake by macrophages in vitro, J. Biol. Chem. 264 (1989) 10749-10755.

[52] K. Paananen, P.T. Kovanen, Proteolysis and fusion of low density lipoprotein particles independently strengthen their binding to exocytosed mast cell granules, J. Biol. Chem. 269 (1994) 2023-2031.

[53] T. Hevonoja, M.O. Pentikäinen, M.T. Hyvönen, P.T. Kovanen, M. Ala-Korpela, Structure of low density lipoprotein (LDL) particles: basis for understanding molecular changes in modified LDL, Biochim. Biophys. Acta 1488 (2000) $189-210$.

[54] J.R. Guyton, K.F. Klemp, The lipid-rich core region of human atherosclerotic fibrous plaques. Prevalence of small lipid droplets and vesicles by electron microscopy, Am. J. Pathol. 134 (1989) 705-717. 